\title{
Secondary positrons and electrons observed by PAMELA spectrometer
}

\section{Vladimir Mikhailov ${ }^{1 *}$}

\author{
E. Mocchiutti ${ }^{2}$ on behalf of [PAMELA collaboration $]^{\dagger}$ \\ ${ }^{1}$ National Research Nuclear University MEPHI, Russia \\ ${ }^{2}$ INFN, Sezione di Trieste, Italy \\ E-mail: vvmikhajlov@mephi.ru
}

\begin{abstract}
Precise measurements of electron and positron fluxes in energy range from $80 \mathrm{MeV}$ to several $\mathrm{GeV}$ below the geomagnetic cutoff rigidity were carried out using the PAMELA magnetic spectrometer. The instrument was launched on June 15th 2006 onboard the Resurs-DK satellite on an orbit with 70 degrees inclination and 350-600 $\mathrm{km}$ altitude. PAMELA is collecting data since then. The procedure of trajectories calculations in the geomagnetic filed gives a way to separate stably trapped and albedo components produced in interactions of cosmic ray protons with the residual atmosphere. In this work spatial distributions of secondary electrons and positrons in the near Earth space, including the South Atlantic Anomaly, are presented. Altitudinal, latitudinal, longitudinal and temporal dependencies of the fluxes are discussed. These results are particularly interesting for accurate definition of radiation models on the low Earth orbits.
\end{abstract}

The 34th International Cosmic Ray Conference,

30 July- 6 August, 2015

The Hague, The Netherlands

\footnotetext{
*Speaker.

${ }^{\dagger}$ See full list of authors in attachment.
} 


\section{Introduction}

Secondary particles produced by cosmic ray interactions with residual atmosphere execute their trajectories in the Earth magnetic field. Flux of secondary electrons was first calculated in paper [1]. Namely in this work it was considered process of charged pions production by cosmic ray proton interactions. Pions then decay throw $\pi^{ \pm}->\mu^{ \pm}->e^{ \pm}$chain to electrons and positrons. Part of secondaries with appropriate directions can be trapped by a geomagnetic field. Because production rate is proportional to cosmic ray intensity $I c r$, residual atmosphere density $\rho(h)$ and particle's time of live is $\propto 1 / \rho(h)$ then resulting intensity of secondary particles $J(h) \propto$

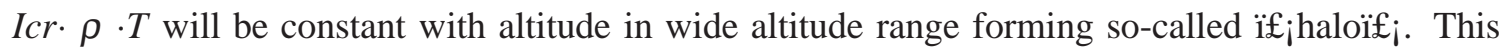
mechanism was verified in several experiments starting from seventieth. Grigorov's simple model did not take into account many features of flux forming. Monte-Carlo simulation also were undertaken to explain results of Maria-2 and AMS-1 experiments. Differences between models and data is still high, about factor 2, perhaps due to differences between modeled and observed conditions. The bactracking procedure which determines the incoming direction of particles before their detection offers new opportunities to compare data with models and allows to determine particles origin [6]. This work presents PAMELA spectrometer measurements of spatial distributions of secondaries electrons and positrons made from 2006 to 2014. The magnetic spectrometer PAMELA was launched onboard the Resurs-DK1 satellite on the 15th of June 2006 and it is continuously taking data till present time. The satellite has a quasi-polar $\left(70^{0}\right.$ inclination) elliptical orbit at an altitudes between 350 and $600 \mathrm{~km}$. Preliminary results of PAMELA observation of secondary electron and positron fluxes near the Earth, which were made in first year of the flight, were reported in paper [2]. At present time about 50TB data were downlinked for analysis during 8 year of the work. Additional accumulated statistics allows to study secondary electrons and positrons generation and propagation in magnetosphere with more details. These results are particularly interesting for more accurate definition of flux model in the Earth magnetosphere.

\section{PAMELA spectrometer}

The instrument consists of a Time-of-Flight system (TOF), an anticoincidence system, a magnetic spectrometer, an electromagnetic calorimeter, a shower tail catching scintillator and a neutron detector. The TOF system gives the main trigger for particle acquisition, measures the absolute value of the particle charge and its flight time while crossing the apparatus (the accuracy is better than $350 \mathrm{psec}$ ). A rigidity is determined by the magnetic spectrometer, composed by a permanent magnet with a magnetic field intensity $0.4 \mathrm{~T}$ and a set of six micro-strip silicon planes. The electron and positron identification is provided by the calorimeter, a series of mini-strip silicon layers interleaved by tungsten planes (16.3 radiation and 0.6 nuclear interaction lengths deep). Particles not cleanly entering the PAMELA acceptance are rejected by the anticoincidence system. Using of the TOF system, the magnetic spectrometer and additional analysis of the calorimeter information allows extracting leptons and measuring their energy (from $50 \mathrm{MeV}$ to several hundred $\mathrm{GeV}$ ) effectively. The acceptance is about $21.6 \mathrm{~cm}^{2} \mathrm{sr}$ [3].

The main axis of PAMELA points to a local zenith. Orbit characteristics allow measuring particles with pitch angles (the angle between the particle velocity and the magnetic field vector) of 


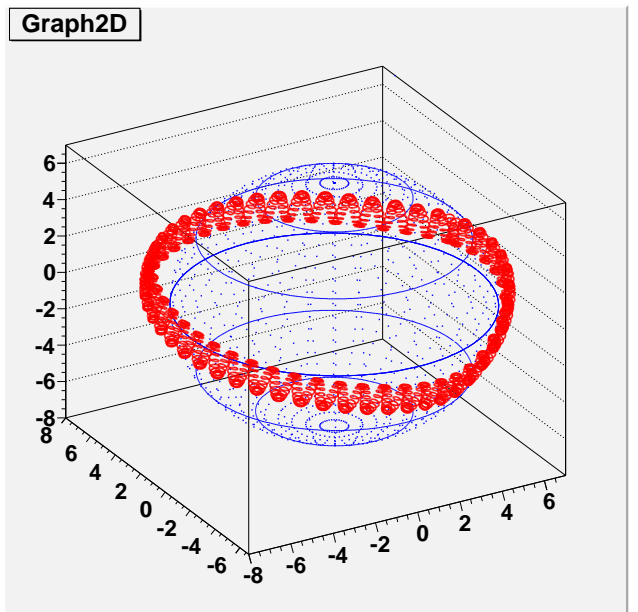

[a] [b]

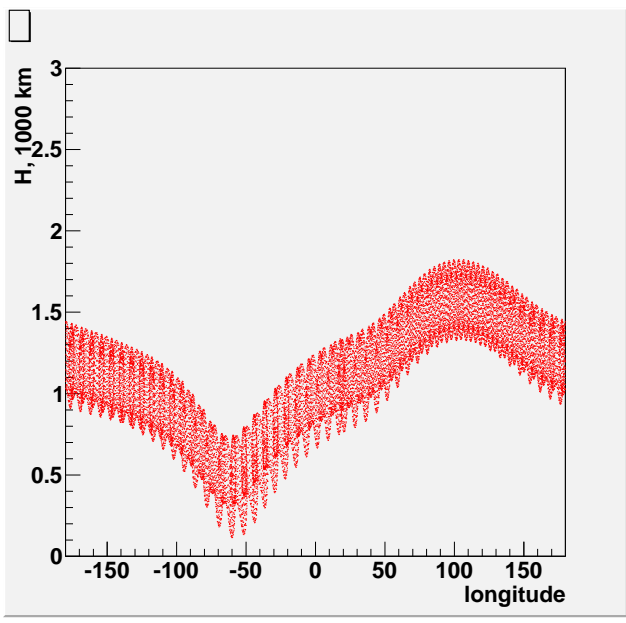

Figure 1: An example of a particle trajectory with energy $1.06 \mathrm{GeV}$ measured by PAMELA. Figure (a) shows 3D view. Figure (b) shows altitude vs longitude.

about $80-90^{\circ}$ in the equatorial region. The angle between the main axis of PAMELA and magnetic field decreases with latitude, so for middle and high latitudes it is possible observe smaller values of pitch angles.

\section{Data Analysis}

For each registered event the following parameters were measured or calculated: the number of tracks and energy losses in the magnetic spectrometer planes; the rigidity and the track length (by fitting the track in the magnetic field); the time of flight. A particle velocity was calculated using the time of flight and the track length. Moreover a set of variables dealing with point of interaction, transversal and longitudinal profiles was calculated using the calorimeter [3].

Electrons and positrons were identified using information about $d E / d x$ energy losses in the spectrometer planes to determine charge $|Z|=1$, shower properties in the electromagnetic calorimeter, particle velocity and a rigidity. The misidentification of protons and pions is the largest source of background. Particle identification based on the calorimeter data can be tuned to rejection power $10^{4}-10^{5}$ for protons and pions, while selecting $>80-90 \%$ of the electrons or positrons over all energy range.

Total accumulated statistic for electrons and positron is about $6 \mathrm{ï}_{j} 10^{6}$ in whole energy range.

Gathering power of the instrument was estimated with Monte-Carlo simulation with official PAMELA Collaboration software [3]. An efficiency of the instrument may change with time and must be taken into account carefully in a data proceeding. It was verified from experimental data itself by using different combination of information from imaging calorimeter, magnetic spectrometer and time of flight system.

Using a geographical coordinates and an orientation of PAMELA as a function of time, the McIlvain geomagnetic coordinates L-shell and B were calculated for every event. For the calculation, the IGRF model(http: //nssdcftp.gsfc.nasa.gov/models/geomagnetic/igrf) of the Earth magnetic field was used. Then backtracking procedure was applied for every identified events to obtain 


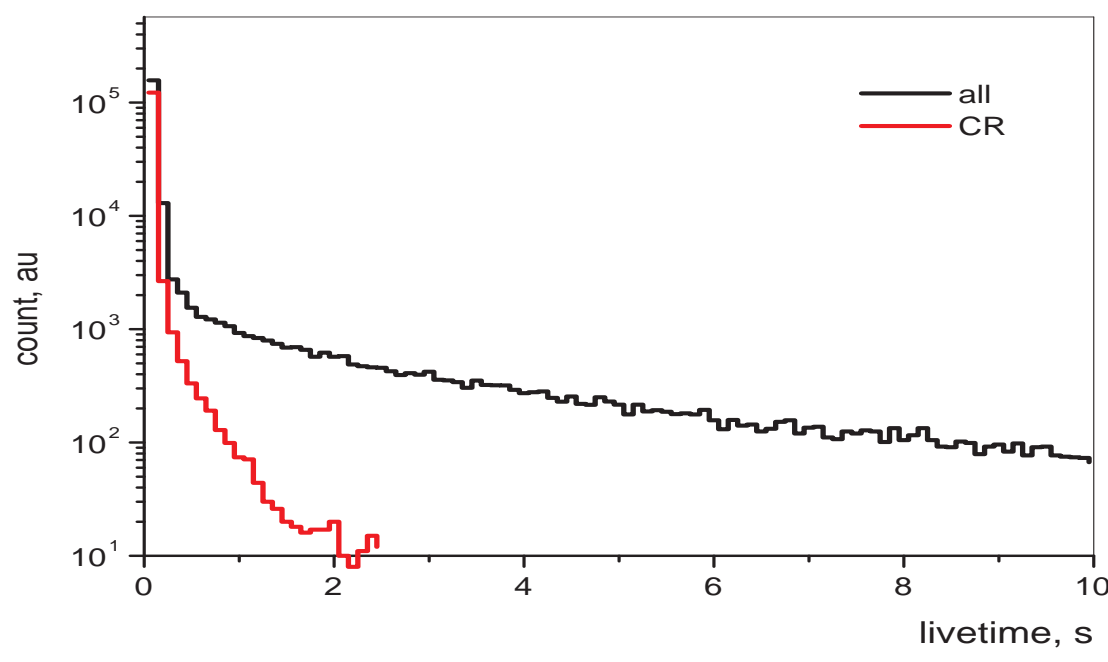

Figure 2: Livetime in magnetosphere for galactic and albedo particles.

trajectory of events up to 10 second before detection and it was stopped if particles touched the Earth atmosphere on $40 \mathrm{~km}$ altutude or escaped magnetosphere. Boundary escaping was chosen to be $20000 \mathrm{~km}$. Trajectory helps to determine particle origin for every individual event. See in figure 1 an example of trajectory of trapped electron with energy about $1 \mathrm{GeV}$.

\section{Results and Discussion}

Due to high accumulated statistic the experimental data show very detail dependence of electron and positron fluxes on particle energy in different spatial regions near the Earth. For electrons and positrons differential spectra, a longitudinal analysis was performed for set of latitudes intervals from equator to pole region [2, 4].These results show features of spectra for different geomagnetic regions due to their origin. Primary particles are observed mainly in polar region and above near equator. Secondary "short lived" component prevails near equator below geomagnetic cut-off at low rigidity and trapped particles can be measured in South Atlantic Anomaly with pitch-angles about 90 degree.

Calculations of particle trajectories in geomagnetic field provides additional information about spatial distribution of fluxes.

The track knowledge give possibility to select particles different origin measured in the same region by track parameters. In figure 2 livetime in magnetosphere is shown for "galactic" and secondary particles. Typically galactic particles reach the orbit of the satellite for time less then 0.1 second. But if electron or positron rigidity is about or slightly less geomagnetic cut-off rigidity this time may increase dramatically because of they executes long chaotic motions before entrance to atmosphere. Secondary albedo have a peak near 0.1 second corresponding reentrant particles, a long tail for quasitrapped component. There is also a part of track with very large livetime which can not be determined by tracking procedure. Figure 3 shows calculated spatial position of trapped 


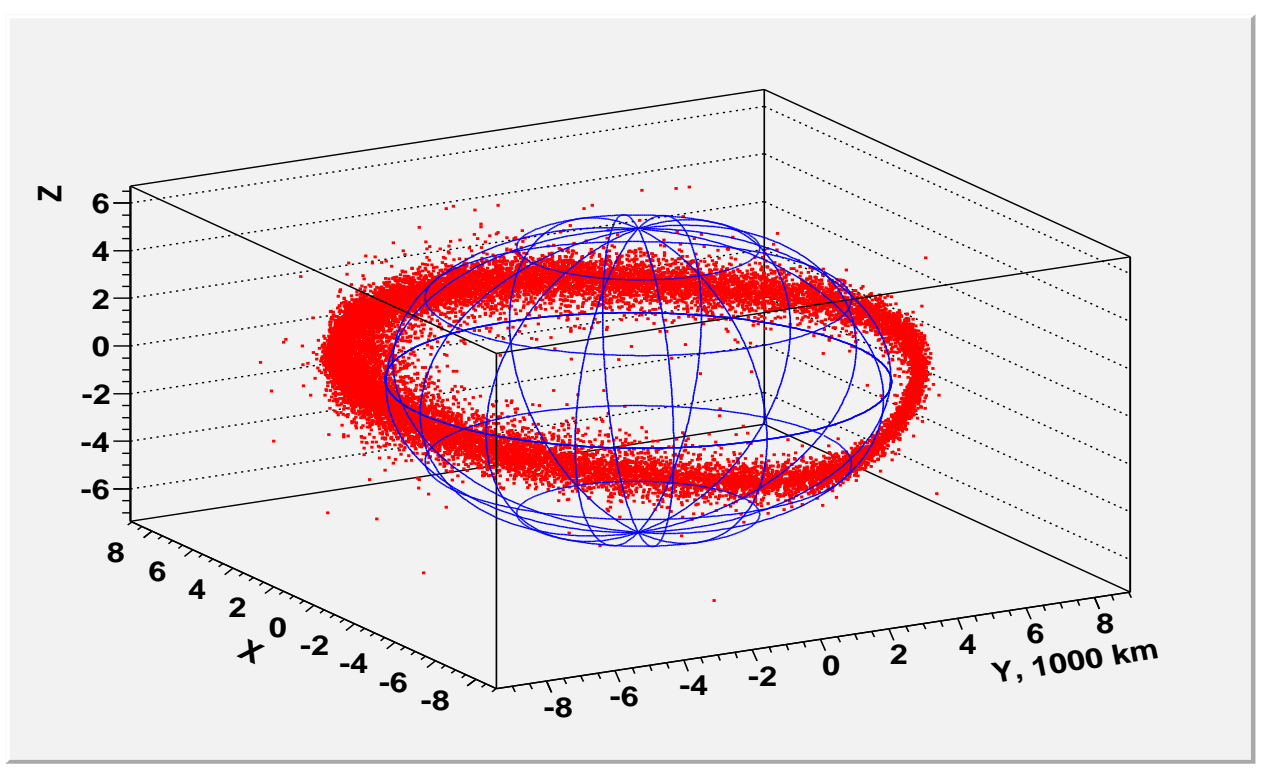

Figure 3: Particles positions 10 second before measurement for trapped positrons and electrons. .

[positrons]

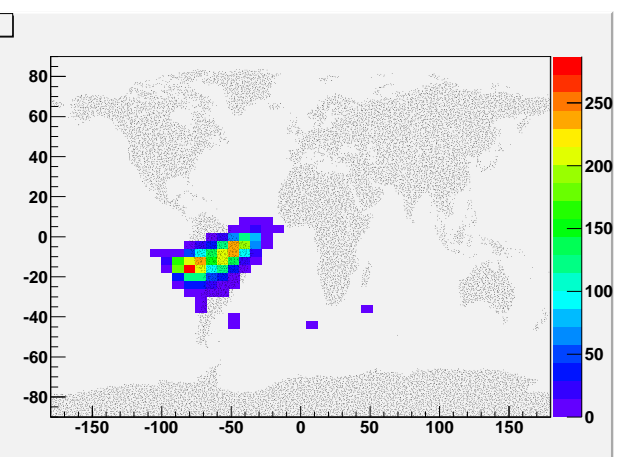

[electrons]

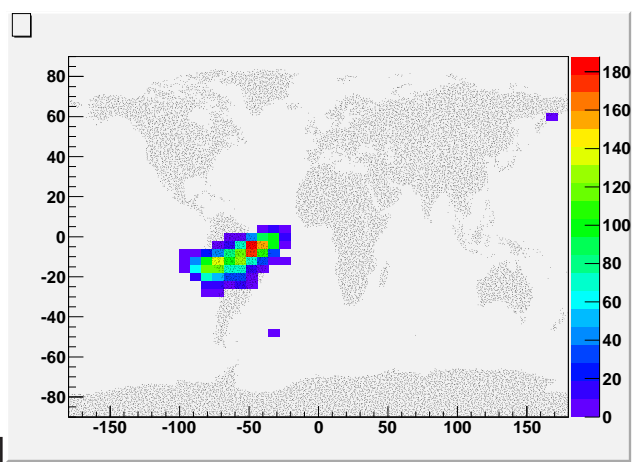

Figure 4: Regions where trapped electrons (right) and positrons (left) were observed by PAMELA.

electrons and positrons they had 10 second before detection. In figure 4 points of measuring of trapped electrons and positrons are shown. Maximum of count is observed at L-shell about 1.18 and it is increasing with local magnetic field B decreasing below 0.215 G. Inside this region positron to electron ratio is maximal near West part of the South Atlantic Anomaly. In agreement with previous analysis [4] positron fraction of very long lived trapped particles is much smaller the simple reentrant albedo one (see figure 5) .

Several attempts were made to estimate secondary electron and positron fluxes, see.e.g. [7]. Nowadays experimental pattern of electron and positron fluxes is much more detail then then theoretically modeled one.

\section{Summary}

Secondary electron and positron fluxes have complex spatial structure caused by geomagnetic field, production cross-section and atmospheric absorption of produced secondaries. Measured 


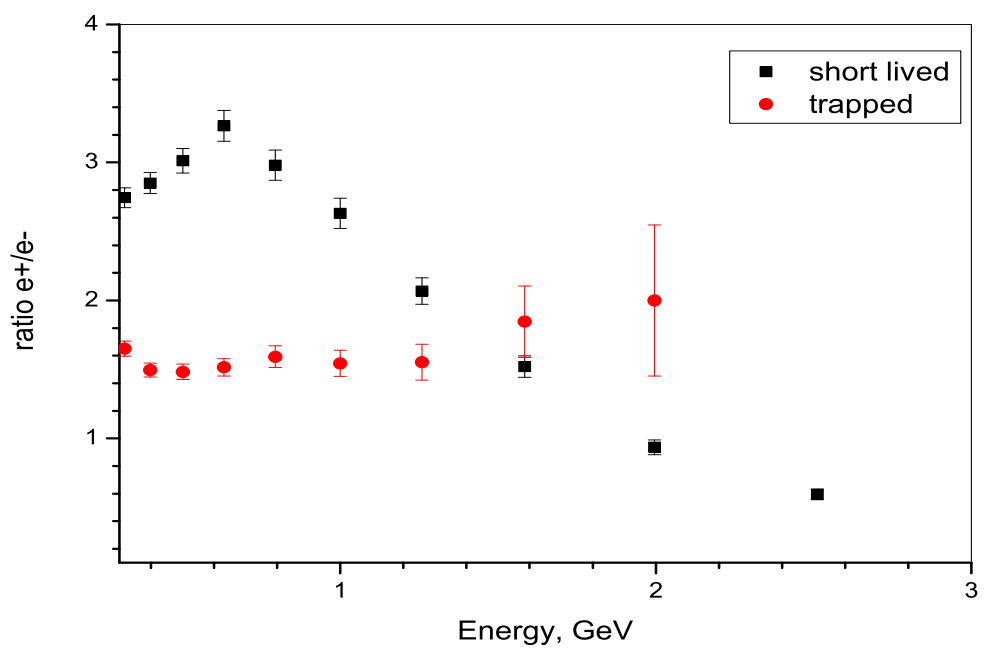

Figure 5: e+/e- ratio positron to electron ratio vs latitude

in the PAMELA experiment electron -positron fluxes and their ratios due to large accumulated statistics allow to study of albedo forming quantitatively and to elaborate an empiric model of secondary particles in the near Earth space.

\section{Acknowlegments}

We acknowledge support from the Italian Space Agency (ASI), Russian Federal Space Agency, Deutsches Zentrum fur Luft- und Raumfahrt (DLR), the Swedish National Space Board, the Swedish Research Council. Russian colleagues also acknowledge partial support from Russian Scientific Foundation (grant 14-12-00373 ).

\section{References}

[1] N.L. Grigorov, Paper of AS USSR, 1977, v. 24, p. 810.

[2] O.Adriani et al.[PAMELA collaboration],Journal of Geophys. Res. 2009,A12218, doi:10.1029/2009JA014660

[3] P. Picozza ,A.M. Galper, G. Castellini et al.[PAMELA collaboration],Astropart. Phys. 2007, v.27: p.296-315

[4] O.Adriani et al.[PAMELA collaboration],Phys.Rep. 2014, v.544, 4, p.323-370, doi: 10.1016/j.physrep.2014.06.003

[5] Alcaraz, J., et al. Phys. Lett. B,2000,v. 484, p.10.

[6] Plyaskin, V. Astropart. Phys.,2008, v.30, p. 18.

[7] Derome, L., M. Buenerd, and Y. Liu , Phys. Lett. B, 2001. 515, 1. 